\title{
Bone metastasis from lung cancer identified by genetic profiling
}

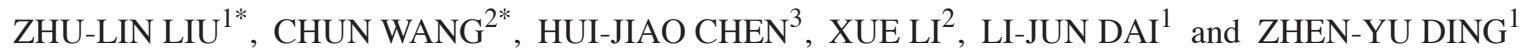 \\ ${ }^{1}$ Department of Thoracic Oncology, Cancer Center, State Key Laboratory of Biotherapy, West China Hospital; \\ Departments of ${ }^{2}$ Endocinology and ${ }^{3}$ Pathology, West China Hospital, West China Medical School, \\ Sichuan University, Chengdu, Sichuan 610041, P.R. China
}

Received May 12,2015; Accepted September 9, 2016

DOI: $10.3892 / \mathrm{ol} .2016 .5458$

\begin{abstract}
Cancer metastasis remains responsible for the vast majority of cases of cancer-related morbidity and mortality. Metastasis, by its definition, is the spread of cancer from the primary site to the distant tissues. Advancing the scientific and clinical understanding of cancer metastasis is a high priority. The prerequisite requirement for pathological consistency may be compromised during metastasis. The present study reports the case of a cancer patient with different pathological types. The patient presented with pain in the neck and right hip, as well as weight loss. He underwent whole-body positron emission tomography-computed tomography, which identified a mass in the lung and abnormal metabolism of the bone. Biopsies of the ilium and lung were performed and he was shown to have lung adenocarcinoma and bone squamous carcinoma. The morphology and immunohistochemical patterns were completely different, while each lesion harbored an identical genetic profile. The bone lesion was identified to be a metastasis from the lung cancer. The patient was prescribed an epithelial growth factor receptor inhibitor, which resulted in a partial response in the lung mass and alleviation of the patient's bone pain. Through this case study, we advocate the importance of using genetic testing in addition to pathological assessment.
\end{abstract}

\section{Introduction}

Cancer metastasis remains responsible for the vast majority of cases of cancer-related morbidity and mortality. Metastasis, by its definition, is the spread of cancer from the primary site to

Correspondence to: Professor Zhen-Yu Ding, Department of Thoracic Oncology, Cancer Center, State Key Laboratory of Biotherapy, West China Hospital, West China Medical School, Sichuan University, 37 GuoXue Lane, Chengdu, Sichuan 610041, P.R. China

E-mail: dingzhenyu@scu.edu.cn

*Contributed equally

Key words: metastasis, genetic profiling, epidermal growth factor receptor the distant tissues. The prerequisite for the establishment of a diagnosis of metastasis is a consistent pathology between the primary site and the metastasis (1). However, to fully endow metastatic potential, cancer cells must accumulate a spectrum of alterations. The pathological consistency may be compromised during this accumulation.

The genomic landscape has been revealed for the most common types of cancer, including lung cancer $(2,3)$. Lung cancer ranks first in both morbidity and mortality worldwide. Its annual incidence in China is estimated to be $\sim 500$ per million people (4). With the progress of genomic technology, lung cancer is considered as a 'disease of the genome'. The gene mutations that confer a selective growth advantage to the tumor cell are called 'driver' mutations (5). The most prominent driver gene in lung cancer is the epidermal growth factor receptor (EGFR) gene (6). Patients haboring EGFR mutation respond markedly to EGFR tyrosine kinase inhibitors (TKI) such as erlotinib (6). The capability of genetic testing expands our armamentarium to recognize occult cancer metastasis.

The present study describes a notable case of lung cancer in which bone metastasis was revealed by genetic profiling, but was not based on pathological analysis.

\section{Case report}

A 44-year-old, non-smoking, male was admitted to West China Hospital (Chengdu, Sichuan, China) on July 16, 2014 with pain in the neck and right hip, and weight loss of 11 pounds over 3 weeks. The physical examination was normal. The patient underwent whole-body positron emission tomography-computed tomography, and a mass in the upper lobe of the left lung and multiple areas of high metabolism in the bone (ilium and first cervical vertebra) were revealed. Magnetic resonance imaging of the neck showed destruction of the Atlas vertebra. The patient then underwent laminoplasty. Biopsies were performed in the right ilium and left lung, and the tissue specimens were fixed in $10 \%$ formaldehyde, embedded in paraffin, and cut into sections $(5-\mu \mathrm{m})$. For immunohistochemical analysis, the sections were incubated for $1 \mathrm{~h}$ at $37^{\circ} \mathrm{C}$ with primary antibodies against cytokeratin (CK) 5/6 (1:100; MAB-0276; Fuzhou Maixin Biotech Co., Ltd., Fuzhou, China), CK7 (1:200; ZM-0071; OriGene Technologies, Inc., Beijing, China), P63 (1:200; CM163C; Biocare Medical, LLC, Concord, CA, USA), NapsinA (1:200; RAB-0639; Fuzhou Maixin Biotech Co., Ltd.) and thyroid 


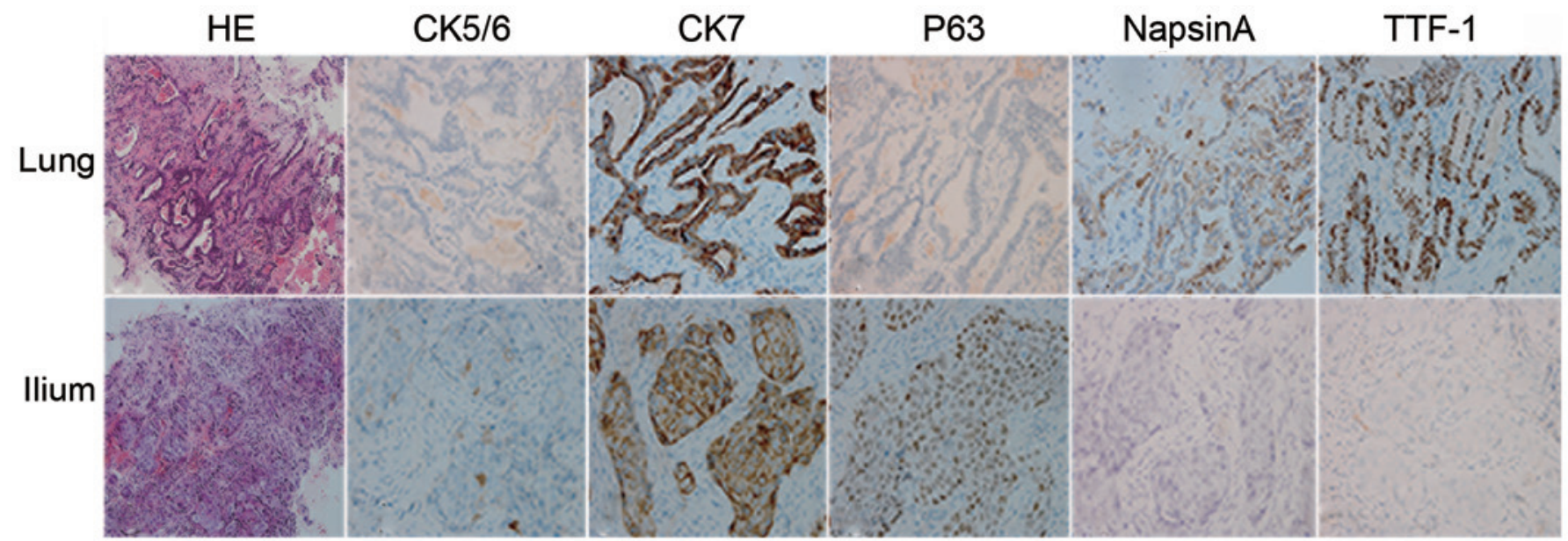

Figure 1. Results of immunohistochemical analysis. Pathological examination revealed a completely different morphological and immunohistological pattern between the lung and bone lesions. While the bone lesion (lower panel) was diagnosed as squamous carcinoma, as shown by HE staining and immunohostochemical analysis, the lung lesion (upper panel) was a typical adenocarcinoma (magnification, $\mathrm{x} 400$ ). HE, hematoxylin and eosin; CK, cytokeratin; TTF, thyroid transcription factor.

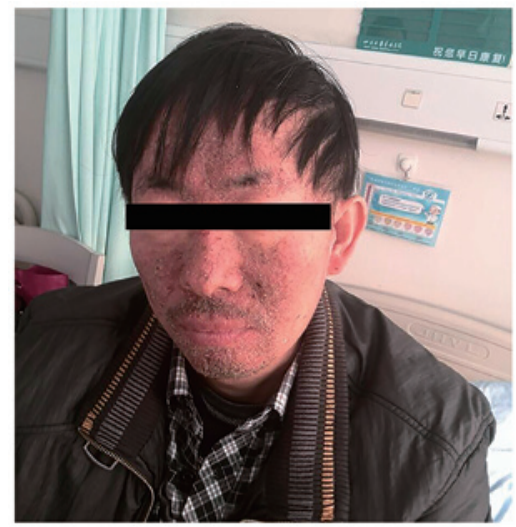

Figure 2. Medicine-related rash on the patient's face. The patient developed a serious acneform rash on the face after being administered erlotinib.
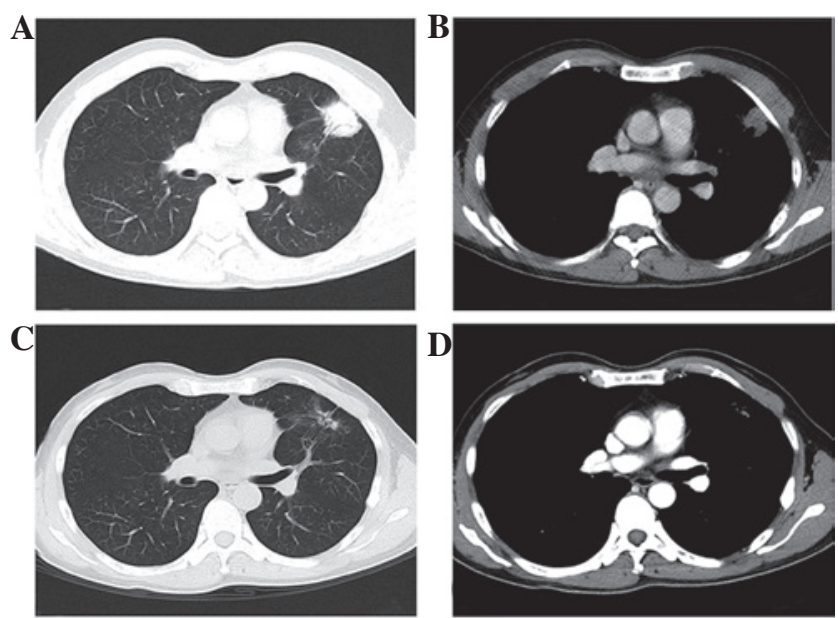

Figure 3. A partial response in the lung. Computed tomography revealed that the tumor was located in the lung (A, lung window; $\mathrm{C}$, mediastinal window). After 3 months of erlotinib therapy, the tumor mass had significantly shrunk (B, lung window; D, mediastinal window).

transcription factor $(1: 200 ; 8 \mathrm{G} 7 \mathrm{G} 3 / 1$; Abcam, Cambridge, MA, USA). Hematoxylin was used for counterstaining. The
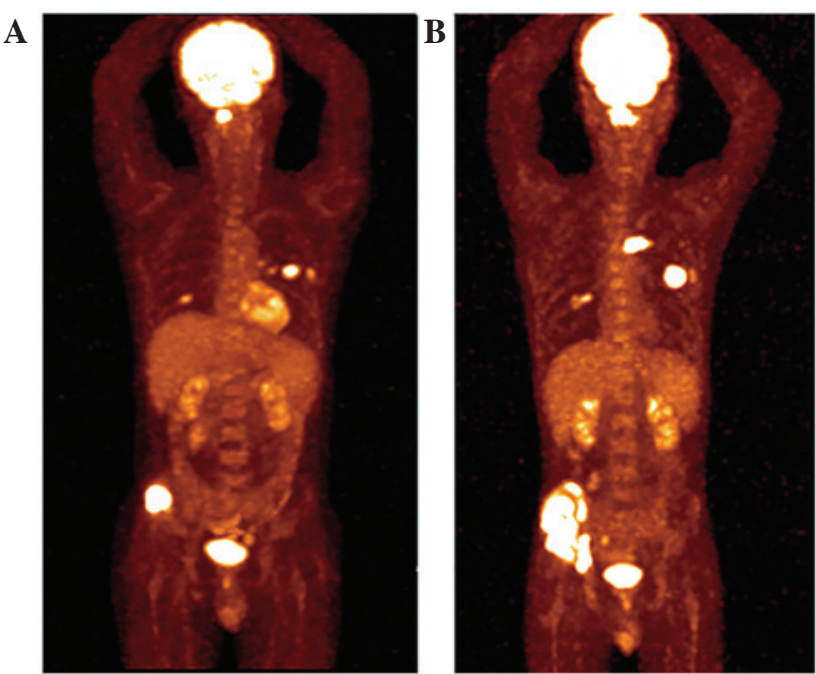

Figure 4. Disease progression after 5 months. The patient exhibited disease progression after 5 months of erlotinib administration, as evidenced by the positon emission tomography-computed tomography scan (A) prior to and (B) following erlotinib treatment.

pathological examination revealed different histological types with different immunohistochemical phenotypes (Fig. 1). However, exactly the same genetic profile between these two lesions confirmed the same entity (Table I). A diagnosis of adenocarcinoma in the left lung with bone metastasis was established (cT3N3M1b, stage IV) (7).

Zoledronic acid $(4 \mathrm{mg})$ was administered each month to prevent severe bone-related events. The patient was also prescribed erlotinib (150 mg, once per day). The medication was well-tolerated, with the exception of a persistent acneform rash on the face (Fig. 2). This TKI treatment achieved a partial response in the primary lung lesion (Fig. 3) and relief of the pain in the right ilium. However, progression of the disease occurred after 6 months of erlotinib treatment (Fig. 4). The patient is currently being treated with doublet chemotherapy consisting of cisplatin $\left(75 \mathrm{mg} / \mathrm{m}^{2}\right.$, day 1$)$ and gemcitabine $(1,000 \mathrm{mg} / \mathrm{m} 2$, days 1 and 8$)$. Chemotherapy is administered 
Table I. Results of genetic testing from the lung and ilium were identical.

\begin{tabular}{lccc}
\hline Region & EGFR & ALK & ROS-1 \\
\hline Lung & G719X+S768I & Wild-type & Wild-type \\
Ilium & G719X+S768I & Wild-type & Wild-type \\
\hline
\end{tabular}

EGFR, epidermal growth factor receptor; ALK, anaplastic lymphoma receptor tyrosine kinase; ROS-1, ROS proto-oncogene 1, receptor tyrosine kinase.

every 3 weeks. Written informed consent was obtained from the patient.

\section{Discussion}

Lung cancer remains the most common cause of cancer-related mortality worldwide (6). Non-small cell lung cancer (NSCLC) constitutes $\sim 80 \%$ of all cases. Targeted therapy has proven its superiority over chemotherapy in patients selected for genetic testing. Therefore, genetic testing has become a focus in lung cancer, and consequently, the genomic landscape has been established by high-throughput sequencing $(2,3)$. The accumulation of genetic data should therefore pose an impact on daily clinical practice.

Traditionally, the present patient would have been diagnosed with different types of cancer in the lung and ilium due to the different pathological types. However, the perfectly matched genetic profile between these two lesions strongly argued that they had arisen from the same origin. This conclusion proposes a challenge to the long-believed metastatic theory of 'pathological consistency'.

We argue that the lesions in the lung and bone belonged to the same disease entity, not only due to their genetic consistency, but also due to their similar responses to a TKI. TKI treatment achieved a significant shrinkage in the lung mass, together with a relief of pain in the bone. The bone lesion was considered non-measurable according to the Response Evaluation Criteria in Solid Tumors (8), so it could not be evaluated precisely.

The same method of deduction has also been proposed by other studies. For example, Sequist et al tracked the genotypic and histological evolution of lung cancers acquiring resistance to EGFR inhibitors (9). The study found that $14 \%(5 / 37)$ of tumors transformed from NSCLC into SCLC. Although these patients developed different histological types of cancer, the same origin of tumor was confirmed by the maintenance of the EGFR mutation. Bloom et al reported that by adapting a micro-array platform, the origin of the tumors could be accurately predicated in $>80 \%$ of histologically similar adenocarcinomas (10). In a review of cancer of unknown primary site, Varadhachary and Raber also exemplified a notable case, in which immunohistochemistry indicated a gastrointestinal origin, while radiographic examination found a solitary lesion in the lung. The microRNA assay finally confirmed a colon-cancer profile (11). Our proposal that the lesions in the current patient had arisen from the same origin was in agreement with these leading-edge reports. 
There is no consensus as to the number of genes used in genetic profiling. In the present case, however, there was high confidence of genetic consistency between the lung and bone lesions. Over $90 \%$ of mutations are located in exon 19 or 21 in the EGFR gene (exon 19 deletion or L858R point mutation). Other rare mutations include G719X, L861Q and S768I (12). The EGFR gene in the present patient contained mutations in two loci: G719X and S768I. Little is known about the incidence of this type of complicated mutation, but it was estimated to be $\sim 0.5 \%$ (as assessed from 12 cases haboring G719X and S768I double mutations from 2,544 patients with the EGFR gene assayed in the past 2 years in West China Hospital). The extreme paucity of this type of mutation almost ruled out the possibility of 'accidental' consistency.

The next question was why the present patient apparently had different types of cancer. Previously, a previous study showed that mixed histological phenotypes were observed in 59 cases from 1,158 lung cancer patients (13). We also reported 21 cases with the mixed form of neuroendocrine tumors from 2,501 primary lung cancer cases (14). Therefore, the heterogeneity in lung cancer was not uncommon as previously believed. Little is known about the origin of the heterogeneity, and theories of hierarchy evolution and multi-clonal origin have been proposed. Notably, the two modes were identified in lung cancer by deep sequencing (15). Neither could be verified in the present patient. The observation of variant histology between primary sites and metastasis was also observed in other case studies (Table II) $(16,17)$. These studies challenged the notion of pathological consistency, but the evidence in support of metastasis was lacking.

In summary, the current study presented a case of advanced lung adenocarcinoma with metastatic squamous carcinoma in the bone. The bone metastasis, although with a different pathological type, was identified by genetic profiling. Through this case report, we advocate the importance of using genetic testing in addition to pathological assessment.

\section{Acknowledgements}

This study was supported by the National Natural Science Foundation of China (grant nos. 82172684 and 81200640).

\section{References}

1. Fidler IJ: The pathogenesis of cancer metastasis: The 'seed and soil' hypothesis revisited. Nat Rev Cancer 3: 453-458, 2003.

2. Cancer Genome Atlas Research Network: Comprehensive genomic characterization of squamous cell lung cancers. Nature 489: 519-525, 2012
3. Cancer Genome Atlas Research Network: Comprehensive molecular profiling of lung adenocarcinoma. Nature 511: 543-550, 2014.

4. Chen W, Zheng R, Baade PD, Zhang S, Zeng H, Bray F, Jemal A Yu XQ and He J: Cancer statistics in China, 2015. CA Cancer J Clin 66: 115-132, 2016.

5. Weinstein IB: Addiction to oncogenes - the Achilles heal of cancer. Science 297: 63-64, 2002.

6. Reck M,Heigener DF, Mok T, Soria JC and Rabe KF: Management of non-small-cell lung cancer: Recent developments. Lancet 382: 709-719, 2013.

7. Goldstraw P, Crowley J, Chansky K, Giroux DJ, Groome PA, Rami-Porta R, Postmus PE, Rusch V and Sobin L; International Association for the Study of Lung Cancer International Staging Committee; Participating Institutions: The IASLC Lung Cancer Staging Project: Proposals for the revision of the TNM stage groupings in the forthcoming (seventh) edition of the TNM classification of malignant tumors. J Thorac Oncol 2: 706-714, 2007.

8. Eisenhauer EA, Therasse P, Bogaerts J, Schwartz LH, Sargent D, Ford R, Dancey J, Arbuck S, Gwyther S, Mooney $\mathrm{M}$, et al: New response evaluation criteria in solid tumours: Revised RECIST guideline (version 1.1). Eur J Cancer 45: 228-247, 2009.

9. Sequist LV, Waltman BA, Dias-Santagata D, Digumarthy S, Turke AB, Fidias P, Bergethon K, Shaw AT, Gettinger S, Cosper AK, et al: Genotypic and histological evolution of lung cancers acquiring resistance to EGFR inhibitors. Sci Transl Med 3: 75ra26, 2011

10. Bloom G, Yang IV, Boulware D, Kwong KY, Coppola D, Eschrich S, Quackenbush J and Yeatman TJ: Multi-platform, multi-site, microarray-based human tumor classification. Am J Pathol 164: 9-16, 2004.

11. Varadhachary GR and Raber MN: Cancer of unknown primary site. N Engl J Med 371: 757-765, 2014.

12. Riely GJ, Politi KA, Miller VA and Pao W: Update on epidermal growth factor receptor mutations in non-small cell lung cancer. Clin Cancer Res 12: 7232-7241, 2006.

13. Ruffini E, Rena O, Oliaro A, Filosso PL, Bongiovanni M, Arslanian A, Papalia E and Maggi G: Lung tumors with mixed histologic pattern. Clinico-pathologic characteristics and prognostic significance. Eur J Cardiothorac Surg 22: 701-707, 2002 .

14. Li DH, Wang C, Chen HJ, Huang H and Ding ZY: Clinical characteristics of the mixed form of neuroendocrine tumor in the lung: A retrospective study in 2501 lung cancer cases. Thorac Cancer 6: 25-30, 2015.

15. de Bruin EC, McGranahan N, Mitter R, Salm M, Wedge DC, Yates L, Jamal-Hanjani M, Shafi S, Murugaesu N, Rowan AJ, et al: Spatial and temporal diversity in genomic instability processes defines lung cancer evolution. Science 346: 251-256, 2014.

16. Glass R, Hukku SR, Gershenhorn B, Alzate J and Tan B: Metastasis of lung adenosquamous carcinoma to meningioma: Case report with literature review. Int J Clin Exp Pathol 6: 2625-2630, 2013.

17. Shelton DA, Rana DN, Holbrook M, Taylor P and Bailey S: Adenosquamous carcinoma of the lung diagnosed by cytology? A diagnostic dilemma. Diagn Cytopathol 40: 830-833, 2012. 